\title{
La formación de conceptos: una comparación entre los enfoques cognitivista y histórico-cultural
}

\author{
Gerardo Ramos Serpa'
}

Adriana López Falcón'

\section{Resumen}

La formación de conceptos es uno de los componentes esenciales tanto del proceso de creación y desarrollo del conocimiento, como de instrucción y aprendizaje en el contexto educacional. Entre las tendencias pedagógicas que estudian esta problemática se encuentran el cognitivismo y el enfoque histórico-cultural. A su vez, la formación de conceptos en el marco del proceso interactivo de enseñanza-aprendizaje tiene lugar en correspondencia con los fundamentos lógico-gnoseológicos que le sirven de base. Se propone como objetivo de este estudio valorar críticamente los fundamentos lógico-gnoseológicos presentes en la comprensión e implementación de tal proceso desde las perspectivas de ambas tendencias, tomando como referencia a dos de los principales representantes de las mismas: David Ausubel y Lev Vygotsky, respectivamente. En el caso de Ausubel, junto a la presencia de aspectos positivos, se aprecian inconsecuencias que hacen que predomine un enfoque lógico-formal que lastra el fundamento científicamente consecuente y el grado de efectividad en la implementación del proceso de formación de conceptos. En el caso de Vygotsky, sin dejar de observar limitaciones en sus fundamentos lógico-gnoseológicos relacionadas con la identificación terminológica en ocasiones de la naturaleza de lo práctico con la del pensamiento o entre conceptos y abstracciones, no obstante se aprecia un mayor grado de cientificidad y consecuencia en las bases de dicho proceso a partir de un análisis lógico dialéctico de contenido basado en lo social y cultural. El estudio de los mismos contribuye a elaborar una comprensión más integral del complejo proceso de formación de conceptos.

\section{Palabras clave}

Formación de conceptos - Cognitivismo - Enfoque históricocultural - Ausubel - Vygotsky.

I- Universidad de Matanzas, Matanzas, Cuba. Contactos: gerardo.ramos@umcc.cu; adriana. lopez@umcc.cu 


\title{
Concept formation: comparing cognitive and cultural-historical approaches
}

\author{
Gerardo Ramos Serpa' \\ Adriana López Falcón'
}

\begin{abstract}
Concept formation is one of the essential components of both knowledge creation and development, and of instruction and learning in the educational context. Cognitivism and the cultural-historical approach are two of the pedagogic trends studying this problem. Concept formation in interactive teaching-learning processes takes place according to its logical-epistemological foundations. This study aims to critically evaluate the logical-epistemological foundations of the understanding and implementation of this process from the perspectives of both trends, referring to two of their main representatives: David Ausubel and Lev S. Vygotsky. In Ausubel's case, along with positive aspects, we have analysed inconsistencies that make a logical-formal approach predominate. Such approach ballasts the scientifically consistent foundation and the grade of effectiveness in the implementation of concept formation processes. As for Vygotsky, while observing limitations in his logical-epistemological foundations related with terminological identification in occasions of the nature of the practical with that of thought, or between concepts and abstractions, we have observed a greater degree of scientificity and consistency in the bases of such process using a dialectical logical analysis of content based on the social and cultural. The study of both authors contributes to elaborating a more integral understanding of the complex process of concept formation.
\end{abstract}

\section{Keywords}

Concept formation - Cognitivism - Cultural-historical approach Ausubel - Vygotsky.

I- Universidad de Matanzas, Matanzas, Cuba. Contactos: gerardo.ramos@umcc.cu; adriana. lopez@umcc.cu 
La formación de conceptos es uno de los componentes esenciales tanto del proceso de creación y desarrollo del conocimiento, como de instrucción y aprendizaje en el contexto educacional.

Recientes trabajos tratan dicha temática a través de sus diferentes aristas, tales como los de Fleury (2006), Bejarano (2013), Farrán (2013), Nava (2013) y Guillen (2014), entre otros.

La formación de conceptos es abordada también desde diversas disciplinas científicas, entre ellas la pedagogía. Aun así, el carácter complejo de su análisis exige un enfoque interdisciplinario que incluye, entre otras perspectivas, la que proviene del tratamiento lógico-gnoselógico de esta cuestión, el cual ayuda a esclarecer los fundamentos más generales sobre los cuales transcurre dicho proceso intelectual.

\section{La formación de conceptos y la educación}

En el contexto educacional se puede constatar que entre las tendencias pedagógicas que le dedican una especial atención a la problemática de la formación de conceptos se encuentran el cognitivismo y el enfoque histórico-cultural. Ambas, desde sus respectivas ópticas científicas, ofrecen un conjunto de apreciaciones que pueden ser provechosamente analizadas y valoradas con el fin de perfeccionar tanto la comprensión misma del proceso de enseñanza-aprendizaje como su implementación.

La base cognoscitiva del proceso educativo en el aula se asienta en el hecho real y objetivo, aunque de carácter ideal, de la fijación y estructuración de los conocimientos científicos en los contenidos pedagógicos a través precisamente de los conceptos; así como en el grado de claridad y precisión con que el alumno se apropia de los mismos y los traduce en habilidades, capacidades y aptitudes.

Es por ello que la formación de conceptos en el marco del proceso interactivo de enseñanza-aprendizaje tiene lugar en correspondencia con los fundamentos lógico-gnoseológicos que le sirven de base. Tales fundamentos se vinculan a la comprensión general del proceso de conocimiento, sus causas y modos de realización, así como con las formas y regularidades a través de las cuales se estructura y se mueve el pensamiento que pretende captar adecuadamente la realidad. Podemos o no ser conscientes de ello, pero tales regularidades se encuentran ahí y funcionan aún sin nuestro conocimiento $\mathrm{o}$ nivel de conciencia y dominio de las mismas. Pero se trata de conocerlas y emplearlas de forma consciente y efectivo en función de las finalidades y condiciones que nos trazamos.

Así, tales regularidades gnoseológicas y lógicas, aun cuando corresponden a los diferentes sujetos en particular, están presentes y operan objetivamente en los procesos intelectuales que se manifiestan en la educación, en tanto esta última supone formar y madurar la capacidad intelectual del individuo de modo consciente y científicamente planeado y ejecutado, a través de las diversas vías y formas en que éste se estructura y desenvuelve, acorde a los diferentes niveles educacionales, sin exclusión de ninguno, desde los grados básicos hasta el posgrado, por supuesto con sus especificidades.

Precisamente esto induce a que los profesores se encuentren conscientes y capacitados para diseñar y desenvolver el proceso de formación y desarrollo de conceptos tomando en consideración tanto los presupuestos teóricos sobre los que el mismo se asienta, así como las variadas prácticas que los llevan a efecto, con sus aciertos y limitaciones en cada caso, sabiendo entenderlas y aprovecharlas.

El problema consiste en que no siempre estos fundamentos lógico-gnoseológicos tienen una base científica sólida, ni son conscientes y consecuentemente asumidos y aplicados. Ello influye decisivamente tanto en la concepción del propio proceso de formación de conceptos que se tenga, como en su implementación pedagógica. 
Por esto, mientras más adecuados $\mathrm{y}$ científicamente válidos sean los fundamentos lógicos y gnoseológicos del proceso de formación de conceptos en este campo, y de modo más consciente y consecuente los mismos sean empleados, en mayor medida se contribuirá a la eficiencia, eficacia y pertinencia del proceso de enseñanza-aprendizaje.

El presente trabajo se propone como objetivo valorar críticamente los fundamentos lógico-gnoseológicos que sirven de base a la comprensión del proceso de formación de conceptos desde los puntos de vista del cognitivismo y del enfoque histórico-cultural. Para ello centraremos nuestra atención en el análisis de dos de los principales representantes de estas tendencias pedagógicas: David Ausubel y Lev Vygotsky, respectivamente. Por supuesto que no es posible hacer una generalización absoluta de que sus posiciones teóricas sean las únicas ni agoten el amplio espectro de estas tendencias, pero de algún modo permiten ilustrar las mismas, además de la impronta que tales autores le impregnan al quehacer de sus seguidores o simpatizantes, vinculados a aquellos que se motivan y ocupan de la trascendente tarea de establecer y desarrollar de modo intencional la capacidad intelectual de los educandos mediante la formación de conceptos.

En primer lugar, nos detendremos en la caracterización de cómo se concibe la formación de conceptos en ambos autores.

\section{La formación de conceptos en David Ausubel}

La concepción ausubeliana del problema se inserta en su teoría del aprendizaje significativo, entendiendo por el mismo la adquisición de significados nuevos por parte del alumno, lo que ocurre cuando las ideas expresadas simbólicamente son relacionadas de modo no arbitrario y sustancial con algún aspecto existente específicamente relevante de los conocimientos estructurados que ya él posee.
Tal aprendizaje se diferencia de esta manera, sustancialmente, del repetitivo o mecánico.

Cabe señalar que aunque la producción científica de Ausubel relacionada con este tema es amplia, incluyendo el abordaje de problemáticas tales como el aprendizaje verbal significativo y su relación con el llamado aprendizaje por recepción, expuesto en su obra original The psychology of meaningful verbal learning (1963); el estudio del desarrollo infantil con sus componentes personológicos y conductuales, lingüísticos, cognitivos y físicos, como expresión de influencias biológicas y sociales (AUSUBEL; SULLIVAN; PENHOS, 1991); el análisis de la adquisición y la retención del conocimiento en su vínculo estrecho con cuestiones tales como los diferentes contextos en que ello puede tener lugar, los nombres de los conceptos y su adquisición a través del aprendizaje representacional significativo $\mathrm{y}$ el incremento del vocabulario de la persona (AUSUBEL, 2000); no obstante, el tratamiento de la cuestión específica de la formación de conceptos aparece de forma madura y clásica como parte de su obra Psicología educativa: un punto de vista cognoscitivo, escrita originalmente en 1968 y ampliada con aportes de varios colaboradores en 1978 (AUSUBEL; NOVAK; HANESIAN, 1997), unido al hecho de que su comprensión de la formación de conceptos ha mantenido los puntos de vista teóricos esenciales que lo caracterizan, por lo que nos detenemos fundamentalmente en esta obra para su apreciación.

Según este autor, para que el aprendizaje sea significativo la tarea de aprendizaje debe ser potencialmente significativa (constituida por un material razonable que pueda relacionarse de manera sustancial y no arbitraria con la estructura cognoscitiva del alumno), y el estudiante debe asumir además una actitud para relacionar el nuevo material de aprendizaje con su estructura existente de conocimientos, en correspondencia con la disposición de contenidos relevantes en dicha estructura (entendida ésta como el contenido 
y la organización totales de las ideas de una persona determinada) (AUSUBEL; NOVAK; HANESIAN, 1997).

A su vez, Ausubel considera que el aprendizaje significativo puede tener lugar de diversos modos, y ser por recepción, si el contenido potencialmente significativo es comprendido o hecho significativo durante el proceso de internalización; o por descubrimiento, si el alumno relaciona intencionada y sustancialmente una proposición potencialmente significativa del planteamiento de un problema a su estructura cognoscitiva con el propósito de obtener una solución que sea susceptible de ser relacionada significativamente con su estructura cognoscitiva (AUSUBEL; NOVAK; HANESIAN, 1997).

Entre los tipos de aprendizaje por recepción se encuentra, junto al aprendizaje de representaciones y de proposiciones, el aprendizaje o adquisición de conceptos; el que a su vez puede ocurrir en forma de asimilación de conceptos o de formación de conceptos.

En general, la adquisición de conceptos es vista como el aprendizaje del significado de los atributos de criterios del concepto, entendidos estos como aquellos rasgos que sirven para distinguir o identificar al concepto.

La formación de conceptos se entiende como aquel proceso propio fundamentalmente de los años preescolares y de los primeros de la escuela primaria, donde los

[...] conceptos cotidianos (primarios) más simples y perceptiblemente fundamentados se adquieren relacionando sus atributos de criterio descubiertos con la estructura cognoscitiva después de haber sido relacionados con los muchos ejemplares particulares de los cuales se derivan (AUSUBEL; NOVAK; HANESIAN, 1997, p. 86).

La asimilación de conceptos es interpretada como aquella forma de aprendizaje que ocurre a partir de los últimos años de la escuela primaria en adelante, donde "los atributos de criterio del concepto se presentan, por definición o con base en el contexto, y luego se relacionan directamente con la estructura cognoscitiva del alumno" (AUSUBEL; NOVAK; HANESIAN, 1997, p. 86).

A continuación caracterizaremos con mayor nivel de detalle la concepción de Ausubel acerca de la formación de conceptos.

Ante todo este autor entiende por concepto a los objetos, eventos, situaciones o propiedades que poseen atributos de criterio comunes y que se designan mediante algún símbolo o signo. Considera que es importante y necesario estudiar los conceptos ya que, en su opinión, "el hombre vive en un mundo de conceptos en lugar de objetos" (AUSUBEL; NOVAK; HANESIAN, 1997, p. 105), los cuales permiten percibir la realidad a través de un filtro conceptual, lo cual hace posible la adquisición de ideas abstractas sin experiencia empírico-concreta, los cuales se emplean tanto para categorizar nuevas situaciones dentro de campos de conocimientos existentes como para servir de postes de afianzamiento a la asimilación y el descubrimiento de conocimientos nuevos.

Para él, el proceso específico de formación de conceptos se caracteriza por el hecho de ser espontáneo, sin guía, de carácter inductivo, donde los atributos de criterio del concepto se adquieren a través de la experiencia directa, transitando por etapas sucesivas de generación de hipótesis, comprobación y por último generalización de las mismas. Aunque, como se ha dicho, este es un proceso que ocurre principalmente en el niño preescolar, considera que puede darse en cualquier edad, lo que amplía el espectro de su presencia y significación en el proceso educacional (AUSUBEL; NOVAK; HANESIAN, 1997, 1997).

Ausubel diferencia entre nombrar conceptos(fenómenovinculadoal establecimiento de equivalencias representativas entre símbolos de primer orden e imágenes concretas) y formar conceptos (proceso asociado a que la palabra comienza a representar significados e ideas genéricas fijados en contenidos cognoscitivos más abstractos y generalizados). Por esto el proceso 
de formación de conceptos va madurando, de modo tal que según su opinión los atributos de criterio concretos se van reemplazando progresivamente por atributos de naturaleza más abstracta. En todo ello desempeña un papel importante el lenguaje al facilitar, mediante las propiedades de representación de las palabras, los procesos de transformación del pensamiento que aquí intervienen; al mejorar y perfeccionar los significados mediante la verbalización y aumentar así su poder de transferencia (AUSUBEL; NOVAK; HANESIAN, 1997).

En tanto Ausubel considera que en el aula es más importante el aprendizaje significativo por recepción que por descubrimiento, a partir de la diferenciación que él mismo establece, por lo que de aquí se deduce también la trascendencia de la formación de conceptos como uno de los modos de llevar a efecto este aprendizaje por recepción.

\section{La formación de conceptos en Lev Vygotsky}

Veamos seguidamente la comprensión de Vygotsky acerca de la formación de conceptos. Pero, para ello, primero se hace necesario esbozar la concepción histórico-cultural que caracteriza la perspectiva teórica general al interior de la cual este autor concibe y expone su visión acerca de la formación de conceptos.

Vygotsky parte del principio de que la conducta del ser humano no es solamente un producto de la evolución biológica, sino que ante todo ella representa un resultado y se encuentra condicionada por el desarrollo histórico y cultural de la propia humanidad. Concibiendo a la cultura como el producto de la vida social y de la actividad mancomunada de los sujetos; así como la esencia del propio proceso del desarrollo cultural, sintetizada en el planteamiento de que "a través de los demás es que nos convertimos en nosotros mismos" (VYGOTSKY, 1987, p. 160), Vygotsky asume la similitud entre el desarrollo cultural y el desarrollo de las funciones psíquicas superiores del ser humano en tanto formas superiores de actividad. De aquí que precise la peculiaridad distintiva de tales funciones específicas propiamente de lo humano, en relación con el llamado proceso de internalización, esto es, aquel proceso mediante el cual la función externa, social e interpsicológica, se hace interna, intrapsicológica. Para él: "La internalización de las formas culturales del comportamiento incluye la reconstrucción de la actividad psicológica teniendo como base las operaciones con signos" (VYGOTSKY, 1994, p. 75). Así, la naturaleza de los procesos psíquicos del sujeto se expresa en su mediatización a través de los signos, el lenguaje y la cultura como tres factores esenciales que condicionan dichos procesos en su propia naturaleza.

Entendiendo a los signos como aquellos estímulos creados artificialmente por el individuo con el fin de influir sobre la conducta propia o ajena, para él la signación (como proceso de creación y utilización de los signos) representa un nuevo principio de actividad que delimita y especifica la naturaleza de la psiquis humana (VYGOTSKY, 1994).

A partir de aquí, Vygotsky concibe al concepto como una formación cualitativamente nueva que no puede reducirse a los procesos más elementales que caracterizan al desarrollo del intelecto en sus etapas tempranas, es una nueva forma de actividad intelectual y un nuevo modo de conducta donde se tiene conciencia de las propias operaciones intelectuales como resultado de auténticos cambios sustanciales, que abarcan tanto el contenido como la forma del pensamiento. En su criterio ello ocurre de manera verdaderamente plena en la etapa de la adolescencia y lo valora como fenómeno primordial de toda la edad de transición. $\mathrm{Al}$ respecto considera que "la formación de conceptos es justamente el núcleo fundamental que aglutina todos los cambios que se producen en el pensamiento del adolescente" (VYGOTSKY, 1991, p. 59).

La formación de conceptos apreciada en toda su trascendencia como una función 
del crecimiento social y cultural total del adolescente constituye una función psíquica superior mediatizada por aquel signo específico que es precisamente la palabra, la cual es utilizada de manera consciente como un medio para la formación misma de los conceptos.

El proceso de formación de conceptos transita por tres fases fundamentales: la de las imágenes sincréticas, la del pensamiento en complejos y la del pensamiento en conceptos propiamente dicho (VYGOTSKY, 1991).

Aun cuando Vygotsky se opone a la exageración del papel de la percepción visual directa y de las vivencias concretas en el niño, que conduce a plantear que el mismo no sabe pensar de manera abstracta, considera que en esa edad lo que existe son equivalentes funcionales de los conceptos o palabras que nombran clases conocidas o grupos de elementos visuales relacionados entre sí por características visualmente comunes, y no conceptos totalmente formados.

Pese a que Vygotsky habla de conceptos reales del niño, a cuyo estudio relaciona los llamados conceptos espontáneos y científicos, de manera rigurosa y consecuente él considera a la formación de conceptos, y a las fases antes dichas, propias sólo de los conceptos artificiales, abstractos o intelectuales del nivel superior, los cuales están presentes de manera plena en la adolescencia, a los que entonces puede calificar entonces de conceptos verdaderos (VYGOTSKY, 1981).

De aquí que para este autor la formación de conceptos, en tanto eslabón básico de todos los cambios en la psicología del adolescente, hace que todas las demás funciones psíquicas se intelectualicen, reestructuren, integren y supediten a esta función central. En ello radica el trascendente papel de la formación de conceptos en el establecimiento y maduración de la personalidad del sujeto.

Subrayando toda la significación de la formación de conceptos para el adolescente en particular, y en general para el individuo como sujeto social, Vygotsky plantea que gracias a dicho proceso es que llegamos a comprender la realidad, a las demás personas y a nosotros mismos. Para él: "El conocimiento en el verdadero sentido de la palabra, la ciencia, el arte, las diversas esferas de la vida cultural pueden ser correctamente asimiladas tan sólo en conceptos" (VYGOTSKY, 1991, p. 64); por lo que el paso al pensamiento en conceptos le permite al adolescente acceder al mundo de la conciencia social objetiva, de la ideología social, apropiarse de la libertad en tanto conocimiento de la necesidad, y con todo ello formar su personalidad y su concepción del mundo.

\section{Los fundamentos lógico-gnoseológicos de la formación de conceptos en David Ausubel y Lev Vygotsky}

Las concepciones anteriormente expuestas de la naturaleza y el proceso de formación de conceptos, tanto en Ausubel como en Vygotsky, se asientan en fundamentos lógico-gnoseológicos específicos y, en ocasiones, contrapuestos.

En el caso de Ausubel se parte de concebir la relación entre los conceptos y la realidad física de modo tal que los primeros constituyen una versión esquemática (en buen sentido) y selectiva de la segunda, por lo que la realidad conceptual es vista no como una representación caprichosa o ilógica del mundo físico, sino como una reproducción que identifica los aspectos notables e importantes de dicha realidad (AUSUBEL; NOVAK; HANESIAN, 1997).

Este fundamento objetivo del proceso de formación de conceptos se une al reconocimiento de que en el mismo se abstraen las características esenciales de una clase de objetos o acontecimientos, vinculando así los conceptos (al menos en su intención) no a cualesquiera rasgos, sino precisamente a los de carácter esencial. Por ello, Ausubel opina que la realidad objetiva que es denotada por un concepto determina en gran medida la utilidad del mismo, acercándose así (aunque lateral y tímidamente) a la validez 
práctica de tales conceptos (AUSUBEL; NOVAK; HANESIAN, 1997).

Así mismo, Ausubel centra la atención y destaca el papel relevante de los conocimientos que ya posee el sujeto y la necesidad de correlacionar con ellos, en el marco de la estructura cognoscitiva, la adquisición de los nuevos conceptos.

En las anteriores consideraciones se aprecia, no obstante, el intento de alejarse de la concepción del reflejo (entendido de modo activo y creativo) y de la verdad, al considerarse a los conceptos como versiones que identifican o denotan aspectos de la realidad objetiva. Ello se correlaciona con la imprecisión que supone definir a los conceptos como objetos, acontecimientos o situaciones, y no como reproducciones ideales de tales fenómenos.

En ocasiones, Ausubel identifica la existencia real sólo con la existencia objetiva, cuando por ejemplo caracteriza al concepto como "una abstracción o una idea genérica que no existe realmente" (AUSUBEL; NOVAK; HANESIAN, 1997, p. 59), aunque en otros lugares aclara que tal irrealidad de los conceptos se refiere al mundo físico.

En el curso del rechazo a lo que Ausubel llama "excesivo entusiasmo en el aprendizaje por descubrimiento", este autor se opone también a lo que considera una epistemología obsoleta que se basa en la búsqueda de la verdad, en tanto opina que únicamente existen "concepciones humanas de la verdad" (AUSUBEL; NOVAK; HANESIAN, 1997, p. 145). No obstante, si bien es acertado renunciar a alcanzar una verdad final, acabada e inmutable, así como reconocer el lado subjetivo de todo conocimiento verdadero; por otro lado, la hiperbolización del carácter relativo de la verdad, el olvido de su lado absoluto (lo que conduce al relativismo) y su referente objetivo no nos debe llevar a renunciar a la verdad como tal, y mucho menos a dejar de considerar su complejidad contradictoria interna, constituida de lados o aspectos tanto absolutos como relativos, objetivos como subjetivos, todo ello social e históricamente condicionado.
Lo anterior se fundamenta en este autor en el hecho de que en el plano lógico la consideración del significado lógico de un conocimiento no tiene que ver con su validez empírica, lo que en realidad evidencia que Ausubel asume y opera ante todo con una lógica formal, es decir, que se desentiende y no se ocupa del análisis y la comprobación del contenido concreto al que se refieren los conocimientos en general y los conceptos en particular. Cuando Ausubel identifica lo abstracto con lo formal (AUSUBEL; NOVAK; HANESIAN, 1997, p. 209), con ello induce al pensamiento en conceptos a la pérdida de contenido y por lo tanto de validez operativa real.

Pero esto, a su vez, es expresión de la concepción ausubeliana según la cual los conceptos captan los rasgos comunes de las clases de objetos de la realidad (AUSUBEL; NOVAK; HANESIAN, 1997). No obstante, como es conocido, lo esencial no puede ser identificado ni reflejado a través simplemente de lo común; ya que por lo regular si el concepto pretende reproducir lo esencial, ello no se puede lograr a partir de detectar los aspectos repetibles sensorialmente perceptibles, sino sobre todo reflejando la ley interna del movimiento del objeto. Aquí se expresa la confusión entre lo esencial y lo universal (por un lado) y lo repetible y lo común por otro.

La comprensión lógico-formal del proceso de formación de conceptos se complementa con la vinculación del mismo a lo que Ausubel llama idea general de categorización, entendiendo por ello el reconocimiento de que "todo tiene un nombre" (AUSUBEL; NOVAK; HANESIAN, 1997, p. 97), con lo que se desdibujan los procesos de nombrar y conceptualizar, asociados pero diferentes en su naturaleza.

Todo lo anterior se asienta en Ausubel en la visión estrecha, unilateral, que de la práctica posee. Si bien es correcta la comprensión ausubeliana de que la práctica no es una variable de la estructura cognoscitiva sino un factor de carácter social que influye sobre ella; la interpretación de la misma como 
"presentaciones repetidas de la misma tarea de aprendizaje” (AUSUBEL; NOVAK; HANESIAN, 1997, p. 276) manifiesta claramente, primero, su reducción al campo educativo, y segundo, su simplificación y empobrecimiento al restringirla a una actividad repetitiva, limitadamente activa y sustancialmente no creativa.

Resumiendo, Ausubel considera acertadamente que la formación de conceptos está determinada culturalmente, y que dicho proceso va madurando progresivamente mediante el establecimiento de abstracciones de más alto orden, más precisas y diferenciadas, a la vez que tiene lugar la toma de conciencia de las operaciones de conceptualización involucradas. En todo ello el lenguaje desempeña un papel importante como facilitador de la generación de nuevos conceptos, debido al poder de representación de las palabras y a la capacidad de nominar las ideas que serán empleadas en el proceso de conceptualización.

Por otro lado, la distinción ausubeliana entre formación y asimilación de conceptos no deja de ser sumamente convencional, ya que cuando el individuo asimila activamente un concepto, evidentemente el resultado no es especularmente el mismo concepto que se le presentó, sino aquel que lleva la impronta de su peculiar estructura cognoscitiva, intereses, necesidades y fines, es decir, un concepto que es re-hecho y re-formado.

Igualmente, al considerarse que en el aula es el aprendizaje significativo por recepción (y la formación de conceptos dentro de él) el que predomina, en tanto "mecanismo humano por excelencia que se utiliza para adquirir y almacenar la vasta cantidad de ideas e información representada por cualquier campo del conocimiento" (AUSUBEL; NOVAK; HANESIAN, 1997, p. 47), entonces

[...] la mayoría de los individuos descubrirá, autónomamente y de ahí en adelante muy pocos conceptos. Las contribuciones de conceptos más difíciles, hechas al almacén de la cultura, las realizan los miembros más dotados en el curso de generaciones y van quedando a la disposición de todos los demás miembros de la sociedad adecuadamente maduros, que pueden captarlos por asimilación conceptual (AUSUBEL; NOVAK; HANESIAN , 1997, p. 106).

Todo ello, sin dejar de ser cierto, puede conducir al peligro de que la escuela efectivamente se adapte y acepte pasivamente esta situación, y renuncie a la finalidad de promover y formar en la mayoría de los miembros de la sociedad las capacidades no sólo para recepcionar los conocimientos ya hechos, sino para asimilarlos creadoramente y descubrirlos permanentemente. Precisamente, Ausubel considera que la escuela solamente puede contribuir a la realización de la creatividad como capacidad "en aquellos individuos raros que ya posean las potencialidades necesarias" (AUSUBEL; NOVAK; HANESIAN, 1997, p. 509), lo cual frisa con posiciones francamente elitistas que deben ser evitadas.

Pasemos a valorar estos fundamentos lógico-gnoseológicos en la comprensión vygotskyana de la formación de conceptos.

El principio teórico rector en los puntos de vista de Vygotsky lo constituye el historicismo, entendido como el estudio del objeto en su proceso de cambio, en su dinamismo, tanto del pasado al presente como en su proyección futura. De esta forma, aplicado al estudio del pensamiento y del concepto como una de sus formas, el llamado método de los cortes genéticos significa el reconocimiento de que la esencia de cada forma del pensamiento (incluyendo aquí al concepto) se revela únicamente al comprenderla como un momento determinado orgánicamente imprescindible del proceso del desarrollo (VYGOTSKY, 1991), lo que inserta y entiende al pensamiento en su espacio natural de devenir.

Ello es expresión de que Vygotsky asume y emplea de manera consciente el enfoque lógico dialéctico, materialístamente interpretado, en el estudio del devenir de la realidad y de su reflejo 
en el pensamiento humano. Oponiéndose a la psicología y a la pedagogía tradicionales y a su enfoque metafísico del pensamiento, expresado en la concepción cuantitativa que interpreta la naturaleza de las formas superiores de dicho pensamiento diferenciándolas de las formas elementales sólo por el número de nexos asociativos que las componen e interpretando al concepto como aquella forma de pensamiento que se fundamenta en lo familiar, lo común, lo repetible, que obvia lo diferente, hace a Vygotsky concluir que "la descripción de la formación del concepto suministrada por la psicología tradicional que reproduce simplemente el esquema de la lógica formal se encuentra totalmente desligada de la realidad" (VYGOTSKY, 1991, p. 96), lo que subraya no solo su desfiguración teórica sino su pérdida de significación práctico-instrumental.

Su perspectiva teórica en el análisis del proceso de formación de conceptos se concreta en el reconocimiento de que a lo largo del desarrollo cultural de la conducta no se modifica sólo el contenido del pensamiento, sino también sus formas. Así, para él, el contenido nuevo no es un líquido que se echa en un recipiente o forma invariable, sino que un contenido determinado puede ser representado de manera adecuada solamente con ayuda de formas determinadas. La formación del concepto modifica sustancialmente el contenido del pensamiento al revelar los nexos profundos $\mathrm{y}$ esenciales de la realidad, así como las leyes que rigen su dinámica. De igual modo, el principal cambio en la forma del pensamiento motivado por la formación de conceptos lo constituye el hecho de que a partir de aquí el sujeto puede dominar su propio pensamiento lógico, lo cual incide a su vez en los constantes cambios del contenido del pensamiento (VYGOTSKY, 1991).

La formación de los nuevos conceptos ocurre en relación directa con todos los restantes y al aparecer los mismos se insertan en el sistema conceptual anteriormente existente, lo que reordena y perfecciona a dicho sistema, lo que representa una característica relevante de su existencia y funcionamiento.

El desarrollo de los conceptos supone la unidad indisoluble del análisis y la síntesis, expresado en la formación de conceptos a través del reconocimiento de que: "Un concepto emerge solamente cuando los rasgos abstraídos son sintetizados nuevamente y la síntesis abstracta resultante se convierte en el instrumento principal del pensamiento" (VYGOTSKY, 1991, p. 94) y donde la palabra es "usada deliberadamente para dirigir todos los procesos de la formación del concepto avanzado" (AUSUBEL; NOVAK; HANESIAN, 1997, p. 94).

Contraponiéndose a la concepción lógico-formal de la psicología tradicional que separa el contenido y la forma del pensamiento, basándose en la ley de la proporcionalidad inversa entre el contenido y el volumen del mismo (lo cual condena inevitablemente al concepto a su empobrecimiento y separación cada vez mayor de la realidad), Vygotsky esclarece la naturaleza esencial del concepto al considerar que:

El verdadero concepto es la imagen de una
cosa objetiva en su complejidad. Tan sólo
cuando llegamos a conocer el objeto en
todos sus nexos y relaciones, tan sólo cuando
sintetizamos verbalmente esa diversidad
en una imagen total mediante múltiples
definiciones, surge en nosotros el concepto.
El concepto, según la lógica dialéctica, no
incluye únicamente lo general, sino también
lo singular y lo particular (AUSUBEL;
NOVAK; HANESIAN, 1997, p. 78).

Los fundamentos lógico-gnoseológicos expuestos se asientan en la comprensión y necesidad de la práctica, en tanto actividad material transformadora, objetiva y subjetiva a la vez, como base objetiva del proceso de formación de conceptos. Aun cuando en determinado momento Vygotsky identifica a la actividad práctica con el "pensamiento práctico" o el "intelecto activo" (AUSUBEL; 
NOVAK; HANESIAN, 1997, p. 151), lo cual suprime la diferencia esencial entre el carácter material de la práctica e ideal del pensamiento; no obstante, en su concepción prevalece el reconocimiento de que son los problemas que le plantea la propia vida al adolescente, así como su incorporación activa a la misma, el estímulo principal del desarrollo de las formas superiores del pensamiento, incluyendo el de los conceptos; tomando en consideración a la actividad práctica de los seres humanos (y específicamente la laboral como factor fundamental del desarrollo cultural del individuo y de la sociedad) en toda su complejidad, mediatizada tanto por las herramientas materiales como por las herramientas del pensamiento que contribuyen a las operaciones intelectuales. Con ello Vygotsky dibuja toda la complejidad y multilateralidad de este fenómeno humano peculiar.

A partir de todo lo anterior este autor argumenta el hecho real de que a través del despliegue del proceso de formación de conceptos se va realizando un reflejo con la mayor profundidad y verdad de la realidad, como proceso gradual e infinito de acercamiento de la imagen intelectual del fenómeno a su esencia real, contradictoria y dinámica.

No obstante, en ocasiones Vygotsky no diferencia suficientemente entre pensamiento en conceptos y pensamiento abstracto, entre concepto y abstracción, lo que no permite reconocer adecuadamente a la abstracción como una operación o acción lógica y al concepto como una forma de existencia del pensamiento que representa, a la vez, un producto y una condición de dicha operación.

De igual modo, cuando Vygotsky plantea que: "El adolescente, con la formación de conceptos, entra en una vía de desarrollo que le conducirá más tarde o más temprano a dominar el pensamiento dialéctico" (AUSUBEL; NOVAK; HANESIAN, 1997, p. 68), ofrece una imagen simplificada de dicho proceso ya que no es de manrea automática, ineludible, ni segura o inviolable que el adolescente llega a dominar un pensamiento dialéctico al formar conceptos, ya que en ello toman parte otros factores tales como las propias tareas o actividades que son diseñadas por el profesor y a ejecutar por el alumno, su grado de maduración intelectual, sus motivaciones y permanencia escolar, el entorno familar y social, entre otros.

\section{Consideraciones finales}

Los fundamentos lógico-gnoseológicos específicos que corresponden tanto a la perspectiva cognitivista representada por Ausubel, como al enfoque histórico-cultural expuesto por Vygotsky, nos permiten comprender tanto los aciertos como las limitaciones de ambas maneras de concebir el proceso de formación de conceptos en tanto propuestas a tomar en consideración en el camino complejo y comprometido de perfeccionamiento de la teoría y la práctica educativa, espacio y vía indispensable del proceso de socialización del individuo y de su formación intelectual e integral.

No se trata de rechazar y subvalorar, ni de asumir acríticamente y mimetizar a cualquiera de estas dos posiciones o autores, todo posee su semilla racional, sus fuentes reales y esenciales. Se trata de descubrirlas, de comprenderlas, de concientizarlas, de leer en el fondo y por detrás de las tesis expuestas, y de interiorizar responsablemente las implicaciones prácticas, formativas $\mathrm{y}$ colectivas derivadas de asumir una posición teórica dada.

Es por ello que desde nuestro punto de vista, tanto Ausubel como Vygotsky ofrecen indispensables referentes a tomar en cuenta para entender e implementar en el espacio educacional el complejo y relevante proceso de formación de conceptos.

En este sentido, consideramos importante resaltar diversos aspectos presentes en las aportaciones de ambos autores para lograr y mejorar un adecuado proceso de formación de conceptos en los educandos. Entre ellos se pueden destacar: 
- el aprovechamiento de los conocimientos preexistentes para ello, aunque no tanto de modo individual sino sobre todo en los espacios colectivos en que ello ocurre en el aula y la escuela, potenciados por los colegas de estudio y maestros;

- la preparación del proceso, los métodos y los medios de enseñanza para favorecer el aprendizaje de modo efectivamente significativo;

- tomar en cuenta y propiciar tanto el aprendizaje por recepción como por descubrimiento, incluso en la escuela;

- comprender que la formación de conceptos transcurre a todo lo largo de la vida humana, tanto dentro como fuera de la escuela, reconociendo las variadas formas en que ello tiene lugar según el nivel de maduración intelectual del sujeto y los fines y peculiaridades de la labor cognoscitiva a realizar;

- considerar los factores lingüísticos, sociales y culturales presentes en el proceso de formación de conceptos, no solo como contextos externos sino como elementos activos que operan en y a lo largo del propio proceso de asimilación y creación de conceptos por los sujetos durante toda su existencia;

- la estrecha correlación entre el contenido y la forma del pensamiento en el caso específico de los conceptos, generados en el curso de la actividad social del sujeto, ante todo práctica;

- la necesaria distinción y concreción del proceso de formación de conceptos en los diferentes estadios del desarrollo psíquico, intelectual y educacional del sujeto.

De aquí que las aportaciones tanto de Ausubel como de Vygostky, los énfasis en diferentes momentos y aspectos del complejo proceso de formación de conceptos, de algún modo se complementan y nos ayudan a apropiarnos de una visión más completa e integral de dicho proceso. Ello no significa eclectizar la representación teórica del mismo, precisamente para evitar esto se han subrayado los, en nuestro criterio, inconsecuentes momentos lógico-gnoseológicos que en determinados aspectos ambos autores evidencian. Se trata más bien de saber discernir y apropiarse de los elementos válidos del aporte de ambos como parte del curso del conocimiento con sus momentos absolutos y relativos hacia la completitud y perfeccionamiento en lo referido a esta compleja y a la vez enraizada cuestión humana de los conceptos y su formación.

En sentido general y en relación con la labor instructiva y formativa de los educadores, la adecuada formación de conceptos no debe ser entendida como una cuestión teórica abstracta, desvinculada de la realidad, con sentido y relevancia únicamente en el aula y para la actividad pedagógica, sin significación práctica que rebase el marco individual dentro del cual aparentemente solo tiene lugar en el intrincado y oculto cerebro del individuo. Más que ello, la formación de conceptos constituye el logro de formar acertamente la manera propiamente humana de captar la realidad, que trasciende lo cognoscitivo y llega hasta lo estético, no solo para entenderla sino ante todo para mejorarla en función de los más altos fines humanos y colectivos. En ello participa la preparación del maestro tanto en el contenido técnico a enseñar como en su formación pedagógica y lógico-filosófica, sus motivaciones y compromiso con la labor de educador, su maestría y recursos para formar las habilidades intelectuales de sus educandos, las formas de evaluación que desarrolle, entre otros muchos elementos.

Ella manifiesta una de las funciones más importantes de la escuela como institución social al preparar al ser humano para la vida, al capacitarlo para apropiarse de lo más elevado elaborado y aculumado por la humanidad que le antecedió, además de ponerlo al nivel de su tiempo y de ofrecerle las herramientas intelectuales para transformar su realidad y humanizar su entorno, convirtiéndolo en un ser activo motivado por los intereses de las mayorías, lo que llama la atención también sobre la dimensión ética de la formación de conceptos, no como fenómeno pura o exclusivamente intelectual, sino ante todo y esencialmente humano. 


\section{REFERENCIAS}

AUSUBEL, David. The acquisition and retention of knowledge: a cognitive view. Boston: Kluwer, 2000.

AUSUBEL, David. The psychology of meaningful verbal learning. New York: Grune \& Stratton, 1963.

AUSUBEL, David; NOVAK, J; HANESIAN, H. Psicología educativa: un punto de vista cognoscitivo. México: Trillas, 1997.

AUSUBEL, David; SULLIVAN, E.; PENHOS, J. El desarrollo infantil: aspectos lingüísticos, cognitivos y físicos. México: Paidós, 1991.

BEJARANO, V. La formación de conceptos científicos en los proyectos pedagógicos de aula. Universidad de Manizalez, 2013. Disponible en: <http://ridum.umanizales.edu.co:8080/jspui/handle/6789/477/>. Acceso en: 10 jan. 2014.

FARRAN, R. La necesidad de una consistencia lógica para la formación de conceptos en la filosofía post-estructuralista. 2013. Disponible en: <http://mylibrary.mediu.edu.my:8181/xmlui/handle/123456789/7804>. Acceso en: 22 ago. 2013.

FLEURY, E. Lenguaje y formación de conceptos en la enseñanza de las ciencias. Madrid: Antonio Machado, 2006.

GUILLEN, Sh. Formación de conceptos y categorías en psicología del pensamiento. 2014. Disponible en: <http://prezi. com/8da7v-xs3qy3/formacion-de-conceptos-y-categorias-en-psicologia-del-pensam/>. Acceso en: 21 mar. 2014.

NAVA, A. Lógica, procedimientos lógicos y la formación de conceptos científicos. 2013. Disponible en: <http://revistas. udistrital.edu.co/ojs/index.php/vinculos/article/view/4209>. Acceso en: 25 feb. 2014.

WGOTSKY, Lev Semenovich. Historia del desarrollo de las funciones psíquicas superiores: La Habana: Científico-Técnica, 1987.

VYGOTSKY, Lev Semenovich. La formación social de la mente. Sao Paulo: Martins Fontes, 1994.

VYGOTSKY, Lev Semenovich. Obras Escogidas: paidología del adolescente. v. 4. Madrid: Centro de Publicaciones del MEC, 1991.

VYGOTSKY, Lev Semenovich. Pensamiento y lenguaje: La Habana: Revolucionaria, 1981. Cap. 5 y 6.

Recebido en: 17.05.2014

Aprobado en: 10.03.2015

Gerardo Ramos Serpa es doctor en ciencias filosóficas y máster en ciencias de la educación superior por la Universidad de La Habana (Cuba). Profesor del Departamento de Estudio y Desarrollo de la Educación Superior de la Universidad de Matanzas, Cuba.

Adriana López Falcón es máster en ciencias de la educación superior por la Universidad de Matanzas (Cuba). Profesora del Departamento de Estudio y Desarrollo de la Educación Superior de la Universidad de Matanzas, Cuba. 